\title{
Pyrroline-5-carboxylate reductase 1 promotes proliferation and inhibits apoptosis in non-small cell lung cancer
}

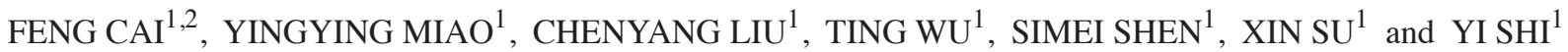 \\ ${ }^{1}$ Department of Respiratory Medicine, Jinling Hospital, Medical School of Nanjing University, Nanjing, Jiangsu 210002; \\ ${ }^{2}$ Department of Respiratory Medicine, Affiliated Hospital of Nantong University, Nantong, Jiangsu 226001, P.R. China
}

Received January 12, 2016; Accepted June 27, 2016

DOI: $10.3892 / \mathrm{ol} .2017 .7400$

\begin{abstract}
Disordered tumor cell metabolism is involved in the process of tumorigenesis. Proline metabolism is of critical importance for tumor cells, and pyrroline-5-carboxylate reductase 1 (PYCR1), a key proline biosynthesis enzyme, has been reported to be overexpressed in prostate cancer and to promote tumor cell growth in breast cancer. The present study investigated the relationship between PYCR1 and non-small cell lung cancer (NSCLC). The results revealed that PYCR1 was overexpressed in NSCLC tumor tissues compared with adjacent normal tissues. High PYCR1 expression was associated with poor prognosis in patients with NSCLC. Following knockdown of PYCR1 by small interfering RNA, cell proliferation was revealed to be significantly inhibited and the cell cycle was arrested, while apoptosis was increased in SPC-A1 and H1703 NSCLC cells. Furthermore, the silencing of PYCR1 resulted in the downregulation of expression of the cell cycle regulator cyclin D1, the regulator of the mitochondrial apoptotic pathway B-cell lymphoma-2, and B-cell lymphoma-extra large. The results of the present study indicated the involvement of PYCR1 in the proliferation and apoptosis of NSCLC. Therefore, PYCR1 may be a novel therapeutic target for inhibiting cell proliferation in lung cancer.
\end{abstract}

Correspondence to: Dr Xin Su or Dr Yi Shi, Department of Respiratory Medicine, Jinling Hospital, Medical School of Nanjing University, 305 East Zhongshan Road, Nanjing, Jiangsu 210002, P.R. China

E-mail: suxinjs@163.com

E-mail: shiyi56@126.com

Abbreviations: NSCLC, non-small cell lung cancer; PYCR1, pyrroline-5-carboxylate reductase 1; $\mathrm{PRODH} / \mathrm{POX}$, proline dehydrogenase/proline oxidase; siRNA, small interfering RNA; Bcl-2, B cell lymphoma-2; TNM, tumor-node-metastasis

Key words: proline biosynthesis, pyrroline-5-carboxylate reductase 1 , non-small cell lung cancer, proliferation, apoptosis

\section{Introduction}

Lung cancer is the leading cause of cancer-associated mortality globally and one of the five top causes of years of potential life lost in East Asia, in 2013. Non-small cell lung cancer (NSCLC) accounts for $80 \%$ of all lung cancers. However, the incidence rates and death rates have declined over the last 20 years $(1,2)$. Due to the advances made in early diagnosis and treatment, the outcome for patients has improved, and this has motivated further research concerning novel targets for the diagnosis and treatment of lung cancer. The disordered metabolism of tumor cells is involved in rapid cell proliferation, maintenance of redox homeostasis and epigenetics $(3,4)$. Interest in this topic has waxed and waned since the observations of Warburg (5). In previous years, multiple oncogenes and tumor suppressors have been linked to the regulation of cancer cell metabolism, making this particular topic one of the most intense areas of cancer research (6).

Proline is one of the most abundant amino acids in the cellular microenvironment. Proline metabolism and synthesis are associated with the tricarboxylic acid cycle, urea cycle and pentose phosphate pathway. Thus, proline metabolism and synthesis are critically important for tumor cells $(7,8)$. Proline dehydrogenase/proline oxidase (PRODH/POX) catalyzes the first step in proline catabolism, and its function in tumors has attracted attention since it was reported to be a P53-induced gene in tumor cell lines $(9,10)$. PRODH/POX is downregulated in multiple types of human tumor, particularly those of the kidney, stomach, colon and rectum. It functions as a mitochondrial tumor suppressor primarily through inhibiting cell proliferation, inducing apoptosis and suppressing hypoxia-inducible factor 1 signaling (11). The function of proline synthesis in cancer, however, remains to be comprehensively understood. Pyrroline-5-carboxylate reductase (PYCR) catalyzes the last step of proline synthesis, and three isozymes are encoded by three human genes. A mutation in PYCR1 was described in patients with autosomal-recessive cutis laxa type 2 , indicating a critical function for proline in normal development (12). An association between PYCR1 gene expression and breast cancer growth has been reported by a previous study (13). These studies indicate that PYCR1 may participate in the process of tumorigenesis.

The aim of the present study was to investigate the function of PYCR1 in lung cancer. PYCR1 expression was detected and 
compared in lung cancer and its adjacent normal lung tissue. Furthermore, the influence of PYCR1 knockdown on cell proliferation, the cell cycle and apoptosis was investigated in order to explore the function of PYCR1 in the tumorigenesis of lung cancer.

\section{Materials and methods}

Patients and tissue specimens. Paired NSCLC samples and adjacent normal tissues were obtained from 28 patients who underwent primary surgical resection to treat NSCLC in the Department of Thoracic Surgery, Jinling Hospital, Nanjing University School of Medicine (Nanjing, China) between July 2014 and September 2014. The specimens were collected during surgery and were kept frozen in liquid nitrogen until RNA and protein extraction. In addition, 62 patients with NSCLC with clinicopathological data and follow-up information (61/62) who underwent surgical resection between June 2007 and November 2008 were enrolled, and their paraffin-embedded specimens were collected from the Department of Pathology, Jinling Hospital, for immunohistochemical staining. Follow-up lasted until February 2015, with a median follow-up period of 84.5 months for living patients (range, 75-94 months). None of these patients received chemotherapy or radiotherapy prior to surgery. The present study was reviewed and approved by the Institutional Review Board of Jinling Hospital, and all patients signed an informed consent form prior to undergoing surgery.

Oncomine $^{\circledR}$ Platform Bioinformatics. The gene search function of Oncomine ${ }^{\circledR}$ Platform (www.oncomine.org) was used to analyze the mRNA expression of PYCR1 in NSCLC tissues relative to their normal controls (14). Gene lists based on fold change were obtained from six NSCLC datasets which were named by the first author and numbers of patient as follows: Selamat (116), Su (66), Stearman (39), Hou (156), Beer (96) and Bhattacharjee (203).

Cell culture. All NSCLC cell lines (A549, SPC-A1, H1703, H1299, PC9, H1915, SK-MES-1) were obtained from the Institute of Biochemistry and Cell Biology of the Chinese Academy of Sciences (Shanghai, China). All cells were cultured in RPMI-1640 medium (Gibco; Thermo Fisher Scientific, Inc., Waltham, MA, USA) except for SPC-A1, which was cultured in Dulbecco's modified Eagle's medium (DMEM; Gibco; Thermo Fisher Scientific, Inc.), supplemented with $10 \%$ heat-inactivated fetal bovine serum (Hyclone; GE Healthcare, Chicago, IL, USA), $100 \mathrm{U} / \mathrm{ml}$ penicillin and $100 \mathrm{mg} / \mathrm{ml}$ streptomycin at $37.8^{\circ} \mathrm{C}$ in a humidified atmosphere containing $5 \% \mathrm{CO}_{2}$.

Reverse-transcription-quantitative polymerase chain reaction (RT-qPCR) analysis. Total RNA was extracted from frozen tissues or cultured cells using TRIzol (Invitrogen; Thermo Fisher Scientific, Inc., Waltham, MA, USA), according to the manufacturer's protocol. Total RNA was reverse transcribed to cDNA using a PrimeScript ${ }^{\mathrm{TM}}$ RT Master Mix (Perfect Real Time) cDNA synthesis kit (Takara Biotechnology Co., Ltd., Dalian, China), according to the manufacturer's protocol. Amplifications were performed in a quantitative real-time PCR machine (Agilent Technologies, Inc., Santa Clara, CA,
USA) using SYBR Premix Ex Taq (Takara Biotechnology Co., Ltd.). The PCR cycling conditions were conducted as follows: $95^{\circ} \mathrm{C}$ for $30 \mathrm{sec}$ followed by 40 cycles of $95^{\circ} \mathrm{C}$ for $5 \mathrm{sec}$ and $60^{\circ} \mathrm{C}$ for $31 \mathrm{sec}$. The primer sequences used were as follows: Human $\beta$-actin forward, 5'-AGCGAGCATCCC CCAAAGTT-3'; and reverse, 5'-GGGCACGAAGGCTCA TCATT-3' (15); human PYCR1 forward, 5'-ACACCCCAC AACAAGGAGAC-3'; and reverse, 5'-CTGGAGTGTTGG TCATGCAG-3' (16). All samples were loaded in triplicates. Relative mRNA expression levels were compared via the $2^{-\triangle \Delta C q}$ method or log-transformed (17). The data were analyzed using MxPro qPCR software v1.2 (Agilent Technologies, Inc.).

Western blot analysis. Total protein was extracted from frozen tissues or cells using RIPA lysis buffer (Beyotime Institute of Biotechnology, Haimen, China) containing a protease inhibitor cocktail (Roche, Basel, Switzerland), at $4^{\circ} \mathrm{C}$ for $20 \mathrm{~min}$, and centrifuged at $14,000 \mathrm{x} \mathrm{g}$, for $10 \mathrm{~min}$ at $4^{\circ} \mathrm{C}$. A total of $50 \mathrm{mg}$ of protein $(5 \mathrm{mg} / \mu 1$ sourced from the tissues and $2.5 \mathrm{mg} / \mu 1$ sourced from the cells) were separated on $12 \%$ SDS-PAGE and blotted onto polyvinylidene fluoride Immobilon-P membranes (EMD Millipore, Billerica, MA, USA). Following blocking with 5\% milk solution for $2 \mathrm{~h}$ at room temperature, the membranes were incubated with primary antibodies against PYCR1 (cat. no. ab150347; dilution, 1:1,000; Abcam, Cambridge, MA, USA), $\beta$-tubulin (cat. no. 2128; dilution, 1:1,000), cyclin D1 (cat. no. 2978; dilution, 1:1,000), B-cell lymphoma-2 (Bcl-2; cat. no. 2870; dilution, 1:1,000), B-cell lymphoma-extra large (Bcl-xl; cat. no. 2764; dilution, 1:1,000) and BCL2 associated $\mathrm{X}$, apoptosis regulator (Bax; cat. no. 5023; dilution, 1:1,000), all purchased from Cell Signaling Technology, Inc., Danvers, MA, USA, overnight at $4^{\circ} \mathrm{C}$. The following day, membranes were probed with horseradish peroxidase (HRP)-conjugated anti-rabbit IgG secondary antibody (dilution, 1:10,000; cat. no. 7074; Cell Signaling Technology, Inc., Danvers, MA, USA), for $2 \mathrm{~h}$ at room temperature. The bands were detected using an Immobilon Western enhanced chemiluminescence HRP substrate kit (cat. no. WBKLS0500; EMD Millipore).

Immunohistochemistry (IHC). Sections of formalin-fixed and paraffin-embedded specimens (5- $\mu \mathrm{m}$ thick) were deparaffinized with xylene and rehydrated in a descending ethanol series, $100,95,70,50$ and $30 \%$. Heat-induced antigen retrieval was performed by submerging slides in $0.01 \mathrm{~mol} / 1$ sodium citrate buffer ( $\mathrm{pH}$ 6.0), and boiling in a microwave oven for $10 \mathrm{~min}$. The sections were treated with $10 \%$ normal goat serum (cat. no. ZLI-9021; ZSJQ-BIO, Beijing, China) to block non-specific binding, for $30 \mathrm{~min}$ at room temperature. Subsequently, the sections were incubated at $4^{\circ} \mathrm{C}$ overnight with the aforementioned primary rabbit anti-PYCR1 antibody (1:200; Abcam). The following day, slides were incubated with HRP-conjugated anti-rabbit IgG secondary antibody (cat no. PV-9001; ZSJQ-BIO; dilution, 1:50), at room temperature for $30 \mathrm{~min}$. Finally, the sections were stained using 3,3'-diaminobenzidine for $10 \mathrm{sec}-1 \mathrm{~min}$ at room temperature, and counterstained with $0.5 \%$ hematoxylin for $1 \mathrm{sec}$ at room temperature. Subsequently, slides were dehydrated, and mounted.

IHC staining was independently scored by two pathologists from the Department of Pathology, Jinling Hospital, without any prior knowledge of patient characteristics, and any discrepancy 


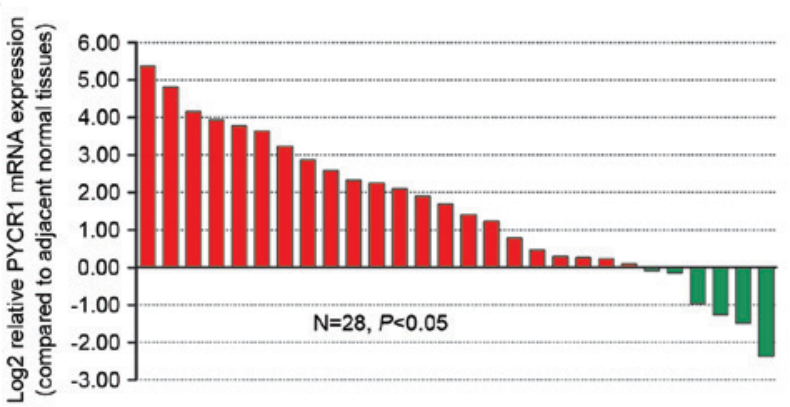

B

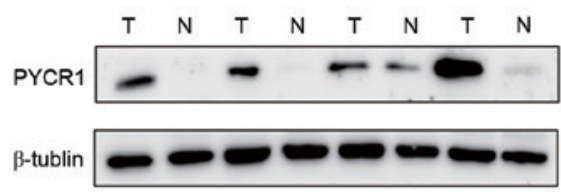

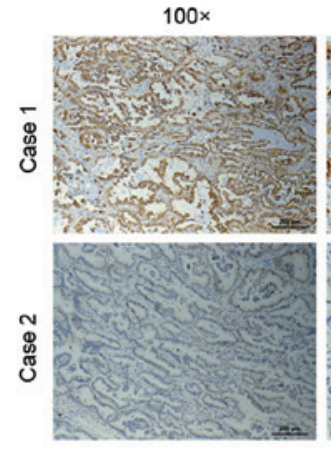

$200 x$

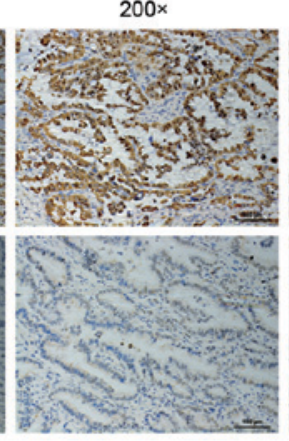

$400 \times$

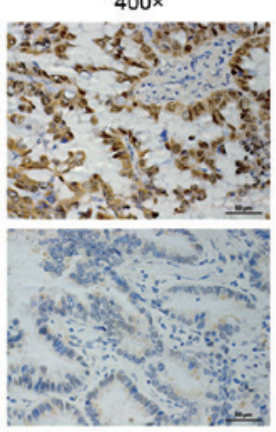

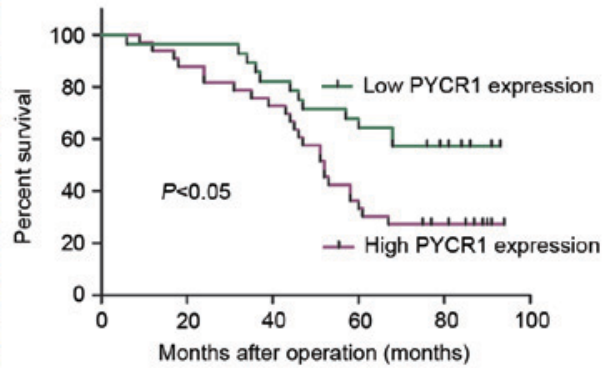

F
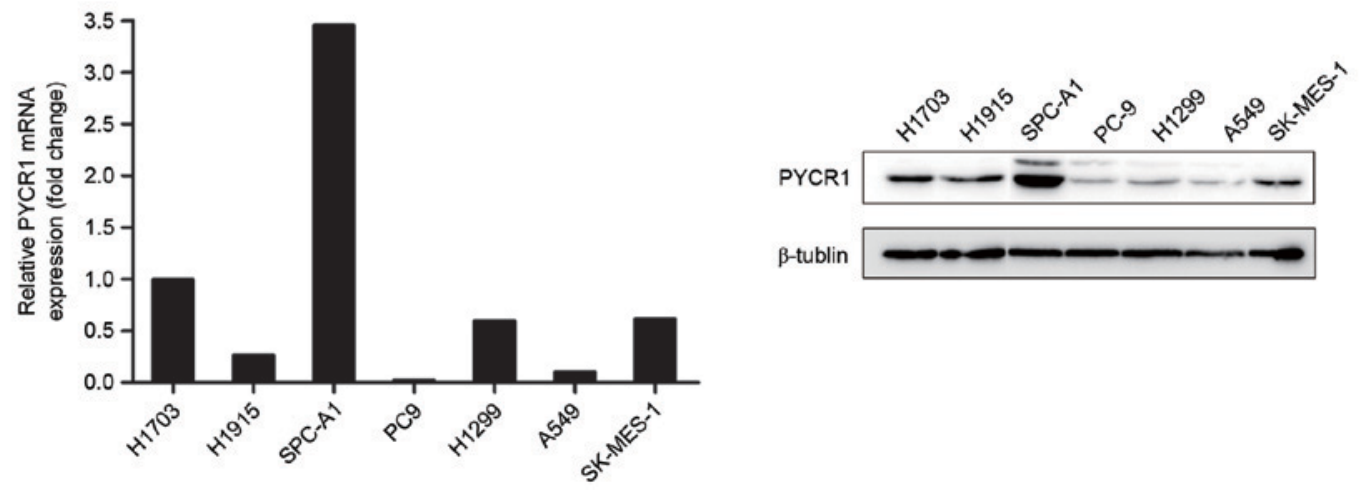

Figure 1. PYCR1 is overexpressed in patients with NSCLC, and high PYCR1 expression is associated with poor prognosis. (A) Log ${ }^{2}$ relative PYCR1 transcript levels in 28 clinical specimens, as analyzed by qPCR. PYCR1 mRNA expression levels were increased in the tumor tissues compared with normal tissues. (B) PYCR1 protein expression levels in the paired NSCLC and normal tissues were detected by western blot. (C) Representative examples of PYCR1 expression in tumor tissues as examined by immunohistochemistry: The upper panel depicts strong PYCR1 staining, and the lower panel weak PYCR1 staining. (D) Kaplan-Meier survival analysis of PYCR1 expression in 61 patients with NSCLC. PYCR1 (E) protein and (F) mRNA transcript expression profiles in 7 NSCLC cell lines were analyzed by qPCR and western blotting, respectively. The mRNA expression levels in NSCLC cell lines were compared with those in H1703. PYCR1, pyrroline-5-carboxylate reductase 1; NSCLC, non small cell lung cancer; qPCR, quantitative polymerase chain reaction; T, Tumor; N, Normal.

was solved by consensus review. Immunostaining was assessed in five fields of view for each sample, under a light microscope (Carl Zeiss AG, Oberkochen, Germany), at x200 magnification. The presence of brown staining within cells was considered to be positive staining for PYCR1. Scores representing the percentage of positive cells were as follows: $0, \leq 5 \% ; 1,6-20 \% ; 2$, $21-50 \%$ and $3, \geq 50 \%$. Staining intensity was scored as follows: 0 , negative staining; 1 , weak staining; 2 , moderate staining; and 3 , strong staining. The product of the two scores was used as the total staining score. In the following analysis, the expression levels were divided into two groups based on the final scores: Low expression $(<3.5)$ and high expression $(\geq 3)$.

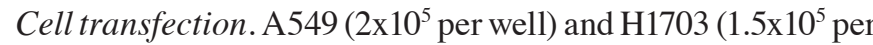
well) cells were cultured for 1 day until they reached $70-90 \%$ confluence. Cells were transfected with PYCR1-specific small interfering (si)RNA or control siRNA using the Lipofectamine 2000 transfection reagent (Invitrogen; Thermo Fisher Scientific, Inc.) according to the manufacturer's protocol. The sequences of the siRNAs used were as follows: Control siRNA sense, 5'-UUCUCCGAACGUGUCACGUTT-3'; and antisense, 5'-ACGUGACACGUUCGGAGA ATT-3'; siRNA-PYCR1 sense, 5'-GCCACAGUUUCUGCUCUCATT-3'; and antisense, 5'-UGAGAGCAGAAACUGUGGCTT-3'.

Cell proliferation assays. Cell proliferation was measured using the CCK-8 cell proliferation kit (Dojindo Molecular Technologies, Inc., Kumamoto, Japan) and 5-ethynyl-2'deoxyuridine (EdU) assay kit (Guangzhou Ribobio Co., Ltd., Guangzhou, China), respectively. For the CCK-8 
assay, siRNA-PYCR1 or control siRNA-transfected cells were cultured in a 96-well plate at a density of 3,000 cells per well. CCK-8 solution $(20 \mu \mathrm{l})$ was added to each well after 0,24 , 48,72 and $96 \mathrm{~h}$. Cells were incubated at $37^{\circ} \mathrm{C}$ for $2 \mathrm{~h}$, and the absorbance of samples was recorded at $450 \mathrm{~nm}$ using an epoch microplate spectrophotometer (BioTek Instruments, Inc., Winooski, VT, USA). All the experiments were performed in triplicate.

For the EdU incorporation assay, dissociated cells were exposed to $50 \mathrm{mM} \mathrm{EdU} \mathrm{(Guangzhou} \mathrm{RiboBio} \mathrm{Co.,} \mathrm{Ltd.)} \mathrm{for} 2 \mathrm{~h}$ at $37^{\circ} \mathrm{C}$. Following fixation with $4 \%$ formaldehyde for $15 \mathrm{~min}$ and permeabilization with $0.5 \%$ Triton X-100 for $10 \mathrm{~min}$ at room temperature, the cells were incubated with $1 \mathrm{X}$ Apollo reaction cocktail (Guangzhou RiboBio Co., Ltd.), $100 \mu \mathrm{l} /$ well for $30 \mathrm{~min}$. Then, the cells were stained with Hoechst 33342 for $30 \mathrm{~min}$ at room temperature and visualized in 3 fields of view/well under a fluorescence microscope (Carl Zeiss). The EdU incorporation rate was expressed as the ratio of EdU-positive cells to total Hoechst 33342-positive cells.

Clone formation assay. SiRNA-PYCR1 or control siRNA-transfected cells were seeded into a 6-well culture plate at a density of 1,500 cells per dish. Cells were maintained in DMEM containing 10\% FBS for 2 weeks and stained with crystal violet for $20 \mathrm{~min}$ at room temperature, for colony counting. The visible colonies were manually counted with the naked eye. All experiments were done in triplicate.

Flow cytometry analysis for cell cycle and apoptosis. Cells, cultured at a density of $1 \times 10^{5} /$ assay, were trypsinized into single cell suspensions and fixed with $70 \%$ ethanol for $30 \mathrm{~min}$ on ice. Then, the cells were stained with propidium iodide (PI) using the CycleTEST TM PLUS DNA reagent kit (BD Biosciences, San Jose, CA, USA) and analyzed using a FACS Calibur flow cytometer (BD Biosciences) equipped with BD CellQuest software v6.1 (BD Biosciences). For apoptosis analysis, cultured cells were harvested by trypsinization and were double stained with Annexin V-fluorescein isothiocyanate/PI apoptosis detection (BD Biosciences) according to the manufacturer's protocol, and analyzed with the aforementioned flow cytometer and software.

Statistical analysis. Statistical evaluation was performed using SPSS 19.0 software (IBM Corp., Armonk, NY, USA). Two-group comparisons were performed using the Student's t-test. $\chi^{2}$ and Fisher's exact tests were used to analyze the associations between PYCR1 expression and clinicopathological features. Kaplan-Meier survival analyses and Cox's proportional hazards models were utilized to determine the association between PYCR1 expression and survival. $\mathrm{P}<0.05$ was considered to indicate a statistically significant difference.

\section{Results}

Elevated PYCR1 expression in NSCLC tissues and cell lines. To investigate the differences between PYCR1 transcript levels between NSCLC tumor tissues and normal tissues, 28 paired samples of fresh frozen normal tissues and tumor tissues were randomly selected, and the mRNA expression levels examined using RT-qPCR. The results revealed that
Table I. Associations between PYCR1 expression and various clinicopathologic features of 62 patients with non small cell lung cancer.

PYCR1 expression

Variable No. of cases High Low P-value

Age at diagnosis, years

\begin{tabular}{lrrrr}
$<60$ & 24 & 13 & 11 & 0.933 \\
$\geq 60$ & 38 & 21 & 17 & \\
Sex & & & & \\
$\quad$ Male & 52 & 30 & 22 & 0.737 \\
Female & 11 & 6 & 5 & \\
Histological type & & & & \\
AC & 27 & 17 & 10 & 0.365 \\
SCC & 25 & 11 & 14 & \\
$\quad$ Other & 10 & 6 & 4 & \\
Tumor differentiation & & & & \\
$\quad$ Well, moderate & 37 & 22 & 15 & 0.374 \\
$\quad$ Poor & 25 & 12 & 13 & \\
Tumor size & & & & \\
$\leq 4$ cm & 35 & 16 & 19 & 0.100 \\
$>4$ cm & 27 & 18 & 9 & \\
Lymph node metastasis & & & & \\
$\quad$ Absent & 37 & 19 & 18 & 0.502 \\
$\quad$ Present & 25 & 15 & 10 & \\
TNM stage & & & & \\
I & 20 & 9 & 11 & 0.560 \\
II & 25 & 15 & 10 & \\
III & 17 & 10 & 7 & \\
\hline
\end{tabular}

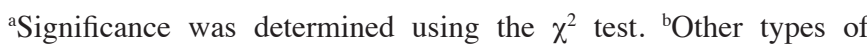
non small cell lung cancer included 8 cases of adenosquamous carcinoma, 1 case of large cell lung cancer and 1 cause of mucoepidermoid carcinoma. PYCR1, pyrroline-5-carboxylate reductase 1; AC, adenocarcinomas; SCC, squamous cell carcinomas; TNM, tumor-node-metastasis.

16/28 patients $(57.1 \%)$ demonstrated higher PYCR1 mRNA expression levels in NSCLC specimens compared with normal tissue specimens ( $\geq 2$-fold change; Fig. 1A). Overexpression of PYCR1 in lung cancers was further supported by data analysis from the Oncomine platform, which revealed higher PYCR1 mRNA expression levels in lung adenocarcinoma tissues, large cell lung carcinoma tissues and squamous cell lung carcinoma tissues than in normal lung tissues. In 4 paired specimens, PYCR1 was overexpressed in all lung tumor samples compared with normal tissues, as detected by western blot (Fig. 1B).

To further study whether PYCR1 protein levels were associated with clinicopathological features and prognosis of patients with NSCLC, 62 paraffin-embedded archived NSCLC tissues were examined using immunohistochemical staining. Representative examples of tumor tissues with either strong or weak staining of PYCR1 are presented in Fig. 1C. The associations between PYCR1 cytoplasmic staining in tumor cells and 
A

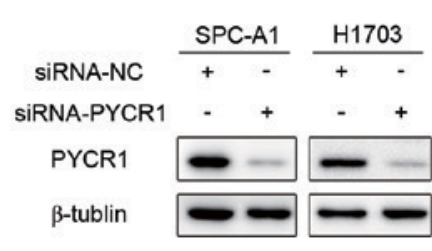

C

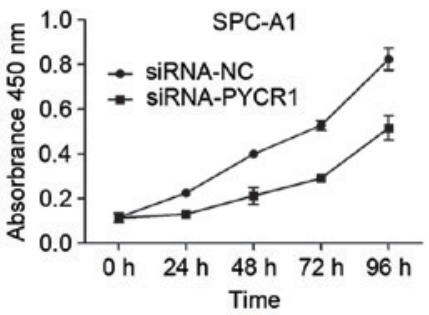

E

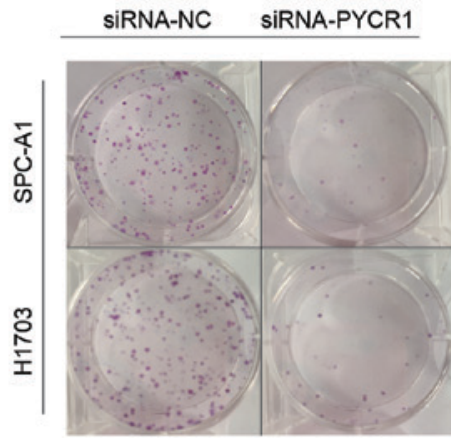

G

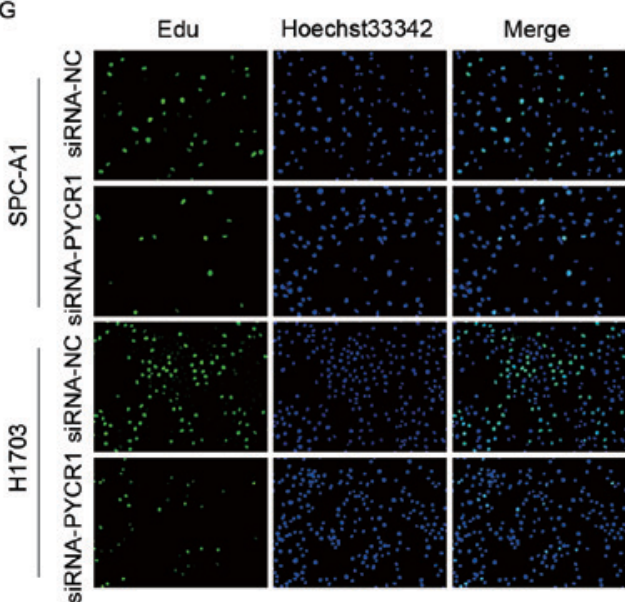

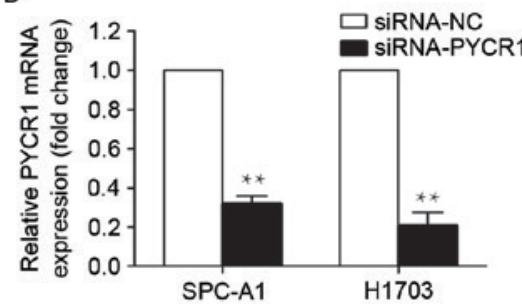

D
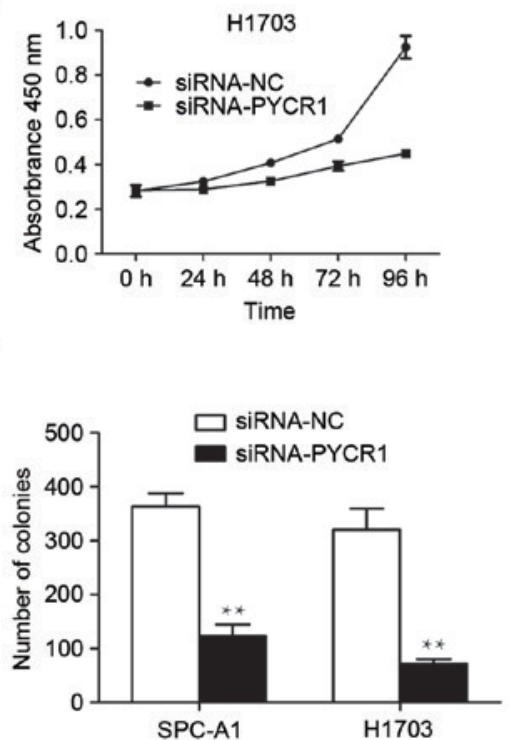

H

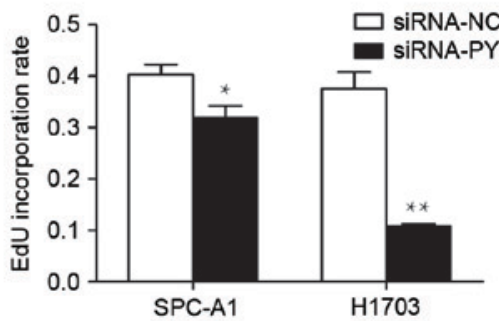

Figure 2. PYCR1 silencing inhibits non small cell lung cancer cell proliferation. (A) Western blot and (B) quantitative polymerase chain reaction analysis of PYCR1 knockdown in SPC-A1 and H1703 cells. Cell proliferation, as detected using Cell Counting Kit-8, demonstrated a significant decrease in (C) SPC-A1 and (D) H1703 cells following PYCR1 silencing. (E) The colony-forming ability of the population was analyzed in siRNA-PYCR1 or siRNA-NC-transfected SPC-A1 and H1703 cells, with (F) quantification. (G) EdU incorporation assays was performed to examine the effect of PYCR1 knockdown on cell proliferation in SPC-A1 and H1703 cells, with (H) quantification. The EdU incorporation rate represents the ratio of EdU-positive cells to total Hoechst 33342-positive cells. The data are expressed as the mean \pm standard error of the mean of three independent experiments. Student's t-test was used to assess significance. ${ }^{*} \mathrm{P}<0.05$ and ${ }^{* *} \mathrm{P}<0.01$ vs. siRNA-NC. PYCR1, pyrroline-5-carboxylate reductase 1; siRNA, small interfering RNA, NC, negative control; EdU, 5-ethynyl-2'deoxyuridine.

the clinicopathological features of the 62 patients with NSCLC were further analyzed (Table I). There was no significant association between PYCR1 expression and any clinicopathological characteristic. However, there was a trend towards higher PYCR1 protein expression in tumor specimens with a larger size $(>4 \mathrm{~cm})$. Clinicopathological characteristics and PYCR1 protein expression were also analyzed using Cox's univariate and multivariate hazard regression models to evaluate whether high PYCR1 expression was an independent risk factor of poor prognosis (Table II). Kaplan-Meier analysis demonstrated that poor overall survival was associated with lymph node metastasis $(\mathrm{P}=0.010)$, tumor-node-metastasis $(\mathrm{TNM})$ stage $(\mathrm{P}=0.010)$ and PYCR1 expression (Fig. 1D; $\mathrm{P}=0.01$ ). For multivariate survival analysis, TNM stage was associated with overall patient survival [hazard ratio (HR): 1.926, 95\% confidence interval (CI): 1.149-3.227, $\mathrm{P}=0.013]$. High PYCR1 protein expression was also revealed to be an independent risk factor for poor prognosis (HR: 2.48, 95\% CI: 1.164-5.286, $\mathrm{P}=0.019$ ). 

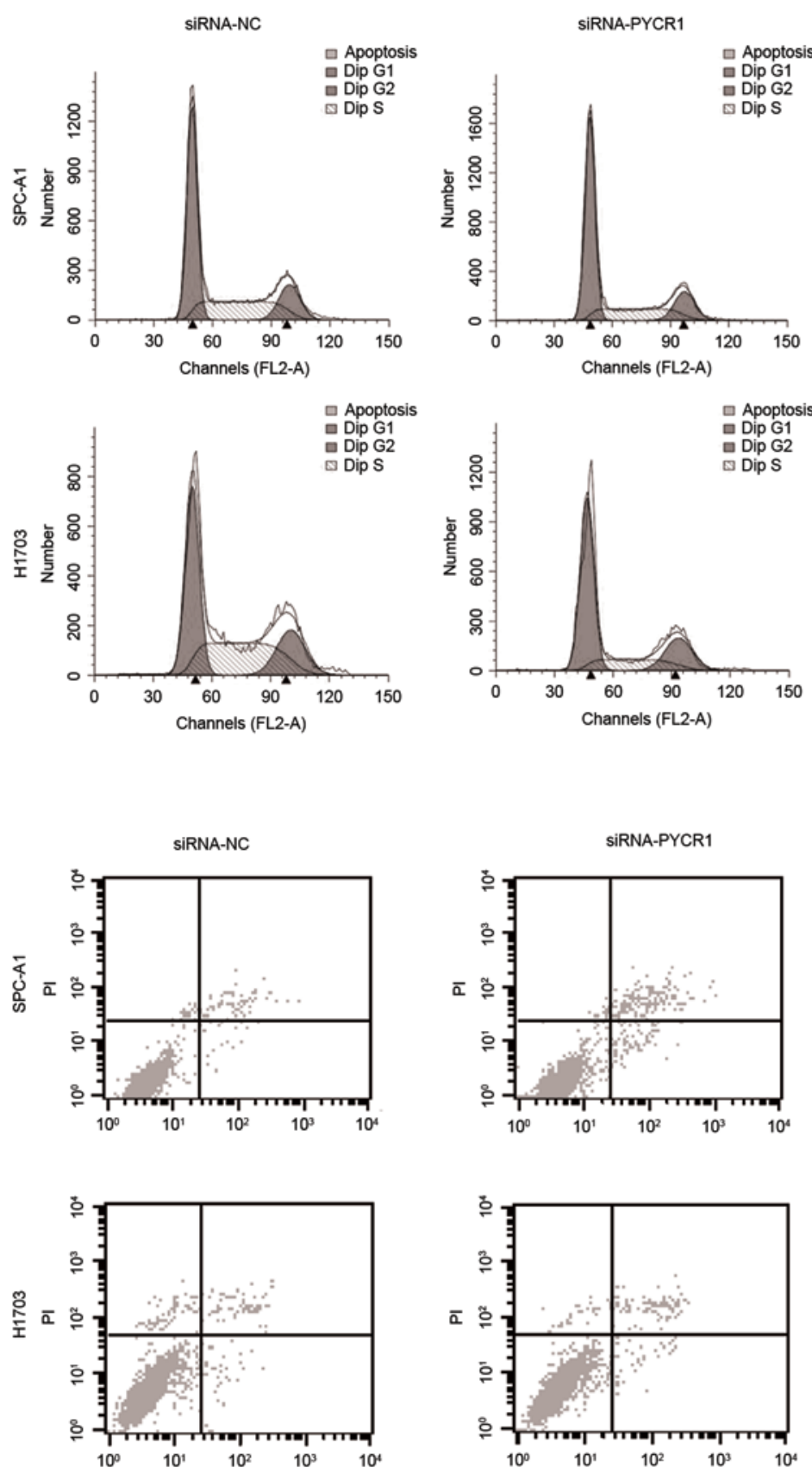
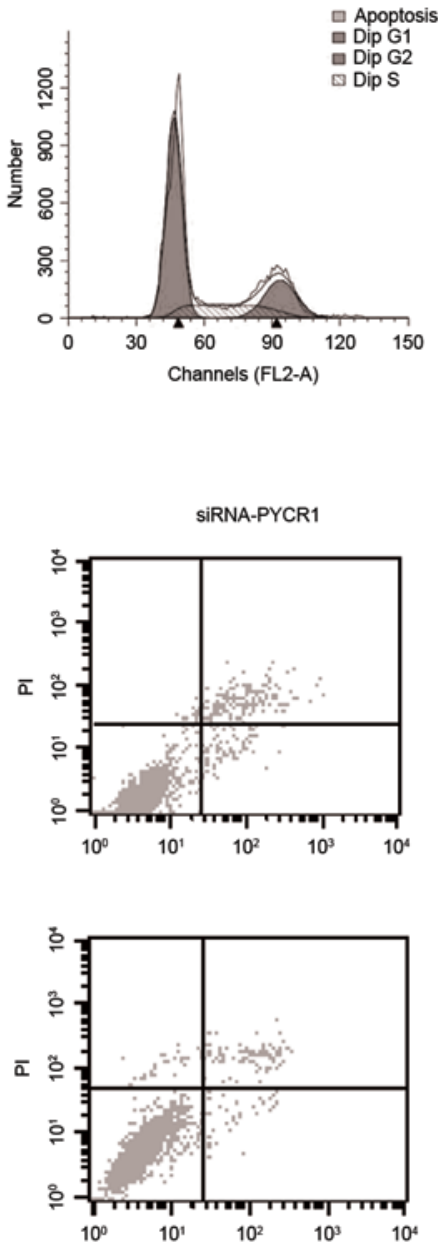
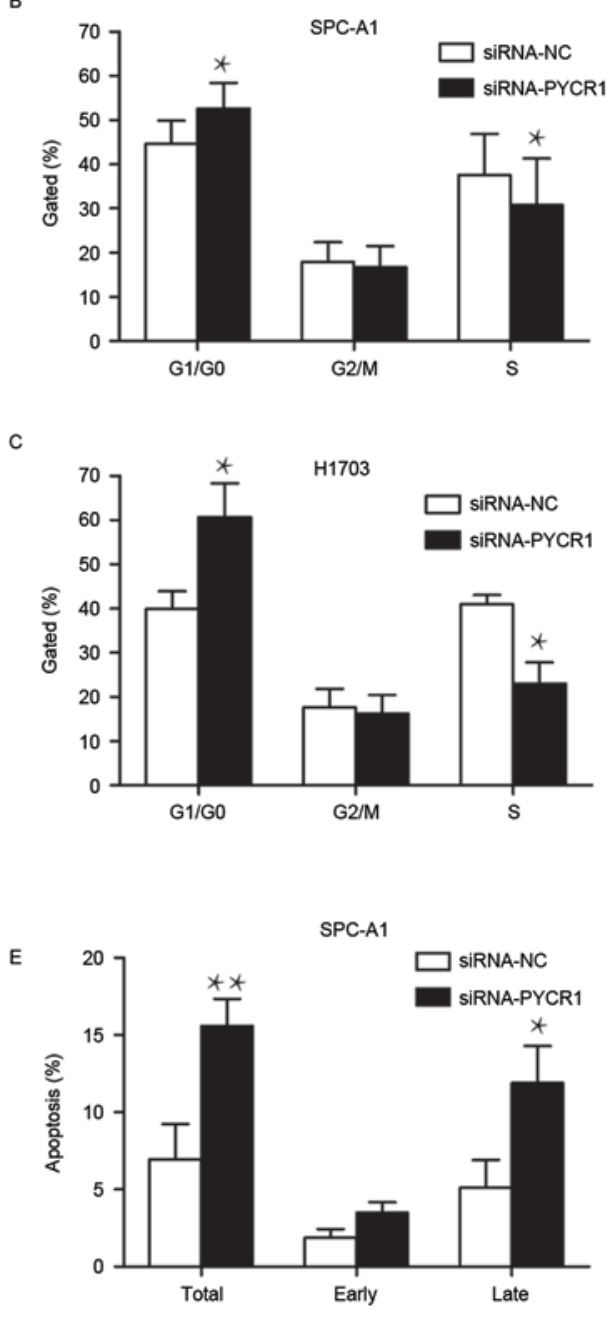

$\mathrm{F}$

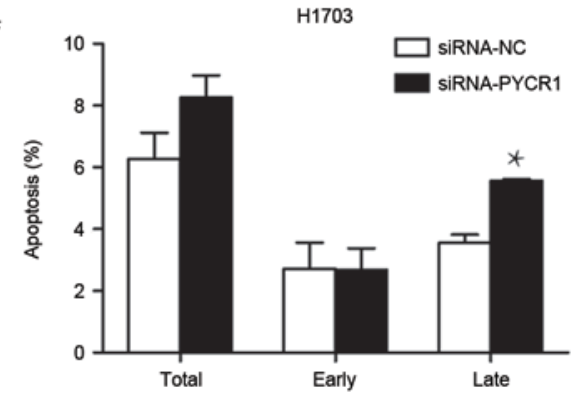

Figure 3. PYCR1 knockdown induces cell cycle arrest and apoptosis. (A) Cell cycle of siRNA-PYCR1 or siRNA-NC-transfected SPC-A1 and H1703 cells was examined by flow cytometry, with quantification for (B) SPC-A1 and (C) H1703 cells. (D) The percentage of apoptotic cells was detected by flow cytometry, with quantification for (E) SPC-A1 and (F) H1703 cells. The data are expressed as the mean \pm standard error of the mean of three independent experiments. Student's t-test was used to assess significance. " $\mathrm{P}<0.05$ and ${ }^{* *} \mathrm{P}<0.01$ vs. siRNA-NC. PYCR1, pyrroline-5-carboxylate reductase 1; siRNA, small interfering RNA, NC, negative control; PI, propidium iodide.

In addition, the mRNA and protein levels of PYCR1 were examined in seven NSCLC cell lines by qPCR and western blotting, respectively (Fig. 1E and F). The results demonstrated that PYCR1 expression level is highly variable in NSCLC cell lines. According to the results, two NSCLC cell lines with high PYCR1 expression (SPC-A1 and H1703) were selected to perform further in vitro investigations.

Knockdown of PYCR1 expression in NSCLC cells suppresses cell proliferation in vitro. As demonstrated by the aforementioned results, tumors with a larger size tended to have relatively high PYCR1 expression, and overexpression of PYCR1 may be involved in survival. Therefore, the influence of PYCR1 expression on the proliferation of human NSCLC cells was studied. SiRNA was used to specifically knock down PYCR1 expression in SPC-A1 and H1703 cells (Fig. 2A and B). PYCR1 knockdown reduced SPC-A1 and H1703 cell proliferation, as assessed using a CCK- 8 cell proliferation kit (Fig. 2C and D). The colony-forming ability of the population was also significantly attenuated following knockdown of 
Table II. Univariate and multivariate analysis of prognostic factors in 61 patients with non small cell lung cancer.

\begin{tabular}{|c|c|c|c|c|c|}
\hline \multirow[b]{2}{*}{ Variable } & \multicolumn{3}{|c|}{ Univariate analysis $^{\mathrm{a}}$} & \multicolumn{2}{|c|}{ Multivariate analysis ${ }^{\mathrm{b}}$} \\
\hline & No. of cases & Mean survival (months) & P-value & HR $(95 \% \mathrm{CI})$ & P-value \\
\hline Age at diagnosis, years & & & 0.794 & & \\
\hline$<60$ & 24 & 64.04 & & & \\
\hline$\geq 60$ & 37 & 62.78 & & & \\
\hline Sex & & & 0.129 & & \\
\hline Male & 51 & 65.44 & & & \\
\hline Female & 10 & 51.50 & & & \\
\hline Histological type & & & 0.447 & & \\
\hline $\mathrm{AC}$ & 27 & 65.60 & & & \\
\hline $\mathrm{SCC}$ & 24 & 63.63 & & & \\
\hline Other ${ }^{c}$ & 10 & 54.70 & & & \\
\hline Tumor differentiation & & & 0.445 & & \\
\hline Well, moderate & 36 & 60.72 & & & \\
\hline Poor & 25 & 66.96 & & & \\
\hline Tumor size & & & 0.672 & & \\
\hline$\leq 4 \mathrm{~cm}$ & 35 & 62.94 & & & \\
\hline$>4 \mathrm{~cm}$ & 26 & 62.46 & & & \\
\hline Lymph node metastasis & & & 0.010 & $1.060(0.461-2.439)$ & 0.890 \\
\hline Absent & 37 & 70.61 & & & \\
\hline Present & 24 & 52.52 & & & \\
\hline TNM stage & & & 0.010 & $1.926(1.149-3.227)$ & 0.013 \\
\hline $\mathrm{I}$ & 20 & 74.80 & & & \\
\hline II & 24 & 59.17 & & & \\
\hline III & 17 & 53.82 & & & \\
\hline PYCR1 expression & & & 0.011 & $2.480(1.164-5.286)$ & 0.019 \\
\hline High & 33 & 55.49 & & & \\
\hline Low & 28 & 72.25 & & & \\
\hline
\end{tabular}

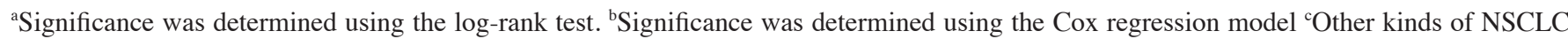
included 8 cases of adenosquamous carcinoma, 1 case of large cell lung cancer and 1 case of mucoepidermoid carcinoma. HR, hazards ratio; CI, confidence interval; PYCR1, pyrroline-5-carboxylate reductase 1; AC, adenocarcinomas; SCC, squamous cell carcinomas; TNM, tumor-node-metastasis.

PYCR1 (Fig. 2E and F). In addition, EdU incorporation assays were performed to explore the effect of PYCR1 knockdown on DNA replication. Following transfection with siRNA-PYCR1, the percentage of EdU-positive cells was decreased by $21 \%$ in SPC-A1 and 71\% in H1703 cells (Fig. 2G and H). These data revealed that PYCR1 is involved in NSCLC cell proliferation.

Silencing of PYCR1 induces cell cycle arrest and apoptosis. To further examine the function of PYCR1 knockdown in cell cycle and apoptosis, the cell cycle and apoptosis were analyzed in siRNA-PYCR1 or control siRNA-transfected SPC-A1 and H1703 cells using flow cytometry. In si-PYCR1-transfected SPC-A1 cells, the proportion of cells in the G1 phase increased by $14.6 \%$ and the proportion of cells in $\mathrm{S}$ phase decreased by $21.6 \%$ (Fig. 3A and B). The altered proportion of G1 and S phase cells was more apparent in siRNA-PYCR1-transfected H1703 cells (Fig. 3A and C). PYCR1 knockdown also induced apoptosis of SPC-A1 cells, in particular late apoptosis (Fig. 3D and E). In H1703 cells, although there was no significant difference in the total apoptosis rate between si-PYCR1-transfected cells and control cells, the late apoptosis rate was increased following PYCR1 silencing (Fig. 3D and F). Taken together, these results demonstrated that the loss of PYCR1 results in the arrest of the cell cycle in the G1 phase and the induction of apoptosis in NSCLC cells.

PYCR1 promotes the cell cycle and inhibits apoptosis through regulating cyclin $\mathrm{Dl}, \mathrm{Bcl}-2$ and $\mathrm{Bcl}$-xl expression. In order to investigate the molecular mechanism underlying the influence of PYCR1 on the cell cycle and apoptosis in NSCLC, key associated regulators were focused on. The cyclin D family are a group of closely related G1 cyclins, and of these cyclin D1 exhibits a more widespread function in human cancers compared with others (18). Thus, it was hypothesized that PYCR1 may promote 

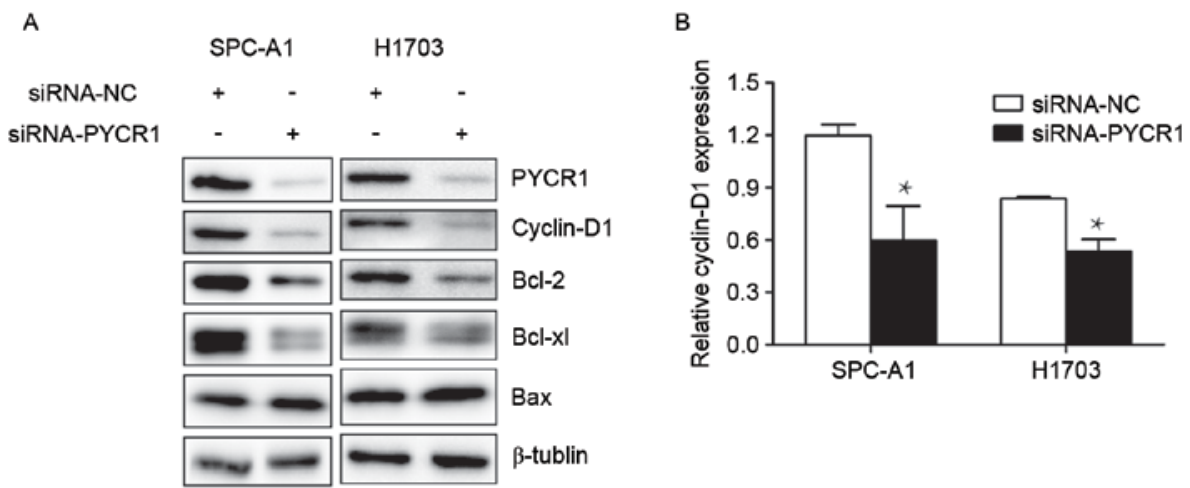

C

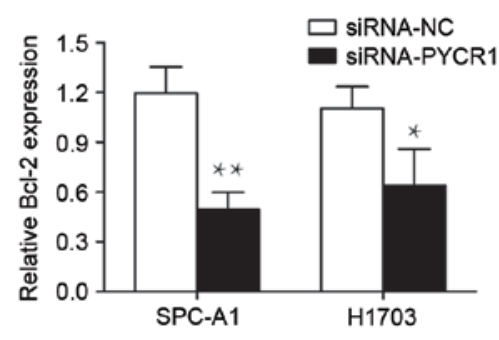

D

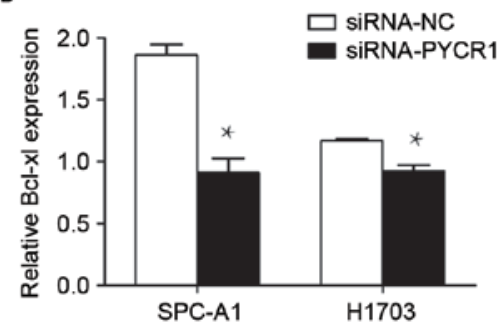

E
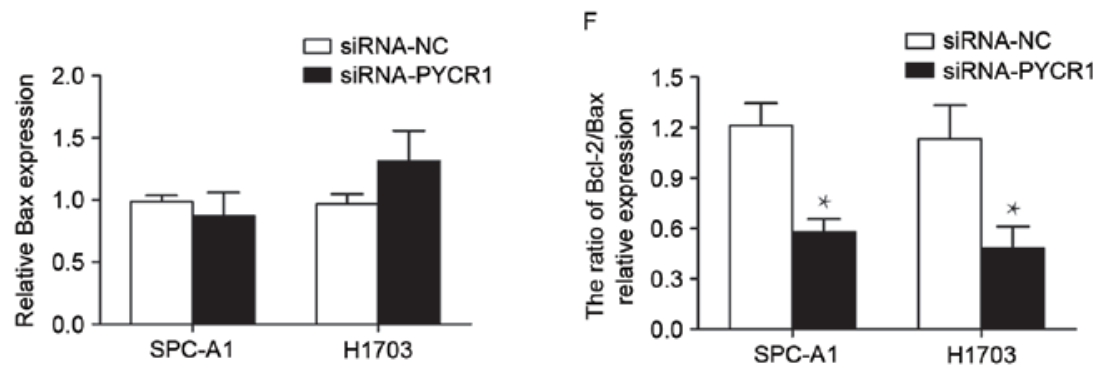

Figure 4. Cyclin D1, Bcl-2 and Bcl-xl were downregulated by the knockdown of PYCR1. (A) Protein expression levels were assessed using western blot analysis following silencing of PYCR1. Quantification was performed for (B) cyclin D1, (C) Bcl-2, (D) Bcl-xl, (E) Bax and (F) the Bcl-2/Bax ratio. $\beta$-tublin was used as an internal control. The data are expressed as the mean \pm standard error of the mean of three independent experiments. Student's t-test was used to assess significance. ${ }^{*} \mathrm{P}<0.05$ and ${ }^{* *} \mathrm{P}<0.01$ vs. siRNA-NC. Bcl-2, B-cell lymphoma-2; Bcl-xl, B-cell lymphoma-extra large; PYCR1, pyrroline-5-carboxylate reductase 1; Bax, BCL2 associated X, apoptosis regulator; siRNA, small interfering RNA; NC, negative control.

the cell cycle from the $\mathrm{G} 1$ phase into the $\mathrm{S}$ phase by regulating the expression of cyclin D1. To test this hypothesis, the expression levels of cyclin D1 following PYCR1 silencing were examined by western blot. The results revealed a significant decrease of cyclin D1 expression in siRNA-PYCR1-transfected SPC-A1 and H1703 cells (Fig. 4A and B).

As for apoptosis, the Bcl-2 family is essential to mitochondrial-controlled apoptosis. As PYCR1 is located on the surface of mitochondrion (19), it was hypothesized that PYCR1 may inhibit apoptosis through its connection to the Bcl-2 family. The results of the present study demonstrated that, following PYCR1 knockdown, Bcl-2 expression levels were significantly decreased in SPC-A1 and H1703 cells (Fig. 4A and C). There was also a decline in $\mathrm{Bcl}-\mathrm{xl}$ expression in the two cell lines (Fig. 4A and D). The Bcl-2/Bax ratio was also decreased, although Bax expression was not affected by the knockdown of PYCR1 (Fig. 4A, E and F).

\section{Discussion}

Disordered glutamine metabolism is involved in lung cancer progression, and has been under intense study to search for promising anticancer therapeutic targets (20). Glutamate is a source of proline synthesis, and this relationship drew the attention of our group to the potential function of proline synthesis in lung cancer. Pyrroline-5-carboxylate (P5C) is an intermediate produced from glutamate by P5C synthase, or from ornithine by ornithine aminotransferase in proline synthesis pathways, and this is then converted to proline through PYCR. Of the three known isozymes of PYCR, PYCR1 is primarily involved in the conversion of glutamate to proline in human melanoma cells (21). A DNA microarray analysis of prostate cancer in 2002 revealed that the expression levels of PYCR1 were significantly increased in prostate cancer (22). The results of the present study demonstrated that PYCR1 is overexpressed in human NSCLC, as detected by RT-qPCR and western blot. Then, by analyzing the clinical significance of PYCR1 expression, it was revealed that in NSCLC tumors with dimensions $>4 \mathrm{~cm}$, PYCR1 expression was higher than those with smaller dimensions (66.7\% vs. $45.7 \%)$. Following multivariate survival analysis, high PYCR1 expression was revealed to be an independent risk factor for poor overall survival, as well as higher TNM stage. 
The involvement of PYCR1 in the proliferation and apoptosis of NSCLC cell lines was also investigated. The results indicated that silencing PYCR1 decreased cell proliferation, resulted in cell cycle arrest at the G1 phase and induced apoptosis. The different medium used for culturing SPC-A1 and H1703 did not alter the effect of PYCR1 on proliferation and apoptosis. H1703 cells were cultured in RPMI-1640 with $0.1 \mathrm{mM}$ proline, while SPC-A1 cells were cultured in DMEM without proline. The similar results in the two cell lines may suggest that the influence of PYCR1 knockdown was not mitigated even with proline at a concentration usually used for essential amino acids $(0.1 \mathrm{mM})$. Possemato et al (13) revealed that knockdown of PYCR1 reduced tumor formation in breast cancer cell lines. A previous study also confirmed the results of the present study: Silencing of aldehyde dehydrogenase 18 family member A1 (P5CS), PYCR1, 2 and L may decrease tumor cell proliferation in several cancer cell lines, including one NSCLC cell line, PC9 (23). However, PYCR1 knockdown did not affect cell cycle and apoptosis in PC9 cells in this study, which is incompatible with the results of the present study. The discordance may be because the expression levels of PYCR1 were decreased in PC9 cells compared with SPC-A1 and H1703 cells, as demonstrated in Fig. 1E and F. The variation in PYCR1 expression across different cell lines may cause differential results in in vitro studies. Repeated studies should be performed in more NSCLC cell lines to confirm the involvement of PYCR1 in the cell cycle and apoptosis in this type of cancer.

The oncogenic transcription factor MYC proto-oncogene, bHLH transcription factor (MYC) is involved in cell proliferation, most notably by targeting G1-specific cyclin-dependent kinases, and it is also associated with apoptosis control $(24,25)$. MYC stimulates mitochondrial biogenesis, which may be important in terms of cell cycle promotion, by helping prepare for cell division (26). MYC promotes proline synthesis from glutamine to proline through increasing the expression of glutaminase, P5CS, and PYCR1. It also inhibits the expression of POX/PRODH and thereby inhibits its function, which is to suppress cell growth and induce apoptosis $(9,27)$. Thus, the proline metabolism enzymes PYCR1 and POX/PRODH may contribute, at least in part, to the effect of MYC on cell growth and apoptosis.

The signaling pathway through which PYCR1 promotes cell proliferation and inhibits apoptosis is still unknown. Cyclin D1 is a key regulator of the G1/S checkpoints and forms a complex with cyclin dependent kinase (CDK) 4/6 (28). Cyclin D1 is also involved in the regulation of apoptosis, and whether it induces or inhibits apoptosis depends on the cell type, its expression level and the growth conditions (29). Due to its involvement in controlling the cell cycle and apoptosis, as well as in lung cancer pathogenesis (30), cyclin D1 expression was detected in siRNA-transfected SPC-A1 and H1703 cells and was revealed to be significantly decreased compared with control cells. In addition, overexpression of cyclin D1 resulted in resistance to cisplatin-mediated apoptosis via the maintenance of Bcl-2 and Bcl-xl protein levels in an ela-myc transgene-expressing pancreatic tumor cell line (31). Bcl-2 family members are critical regulators of the mitochondrial apoptotic pathway through the balance of and competitive dimerization between the anti-apoptotic members, including Bcl-2 and Bcl-xl, and pro-apoptotic members, including Bax and Bcl-2 antagonist/killer 1 (32). High expression of Bcl-2 is associated with an improved outcome in patients with NSCLC with a non-squamous histology, which suggests its involvement in NSCLC (33). In the present study, Bcl-2 and Bcl-xl expression, as well as the ratio of $\mathrm{Bcl}-2 / \mathrm{Bax}$, were revealed to be downregulated following PYCR1 silencing. Taken together, these studies suggested that PYCR1 may promote tumor cell growth by regulating cyclin D1, Bcl-2 and Bcl-xl. Nevertheless, whether the downregulation of $\mathrm{Bcl}-2$ and $\mathrm{Bcl}-\mathrm{xl}$ was directly caused by PYCR1 or through the low expression of cyclin D1 is worth further research.

In conclusion, the results of the present study demonstrated that PYCR1 is overexpressed in NSCLC, and its high expression is an independent risk factor for poor prognosis. PYCR1 may be a key promoter of tumor cell proliferation. In the future, these data should be expanded upon using in vivo models to further elucidate the involvement of PYCR1 in tumorigenesis.

\section{Acknowledgements}

The authors would like to thank Dr Zhongdong Lee from The Department of Cardiothoracic Surgery, Jinling Hosptial, for assisting in collecting specimens.

\section{References}

1. GBD 2013 Mortality and Causes of Death Collaborators: Global, regional, and national age-sex specific all-cause and cause-specific mortality for 240 causes of death, 1990-2013: A systematic analysis for the Global Burden of Disease Study 2013. Lancet 385: 117-171, 2015.

2. Siegel RL, Miller KD and Jemal A: Cancer statistics, 2015. CA Cancer J Clin 65: 5-29, 2015.

3. Vander Heiden MG, Cantley LC and Thompson CB: Understanding the Warburg effect: The metabolic requirements of cell proliferation. Science 324: 1029-1033, 2009.

4. Johnson C, Warmoes MO, Shen X and Locasale JW: Epigenetics and cancer metabolism. Cancer Lett 356: 309-314, 2015.

5. Warburg O: On the origin of cancer cells. Science 123: 309-314, 1956.

6. Cairns RA, Harris IS and Mak TW: Regulation of cancer cell metabolism. Nat Rev Cancer 11: 85-95, 2011.

7. Phang JM and Liu W: Proline metabolism and cancer. Front Biosci (Landmark Ed) 17: 1835-1845, 2012.

8. Phang JM, Liu W, Hancock CN and Fischer JW: Proline metabolism and cancer: Emerging links to glutamine and collagen. Curr Opin Clin Nutr Metab Care 18: 71-77, 2015.

9. Maxwell SA and Davis GE: Differential gene expression in p53-mediated apoptosis-resistant vs. Apoptosis-sensitive tumor cell lines. Proc Natl Acad Sci USA 97: 13009-13014, 2000.

10. Maxwell SA and Rivera A: Proline oxidase induces apoptosis in tumor cells, and its expression is frequently absent or reduced in renal carcinomas. J Biol Chem 278: 9784-9789, 2003.

11. Liu W and Phang JM: Proline dehydrogenase (oxidase) in cancer. Biofactors 38: 398-406, 2012.

12. Guernsey DL, Jiang H, Evans SC, Ferguson M, Matsuoka M, Nightingale M, Rideout AL, Provost S, Bedard K, Orr A, et al: Mutation in pyrroline-5-carboxylate reductase 1 gene in families with cutis laxa type 2. Am J Hum Genet 85: 120-129, 2009.

13. Possemato R, Marks KM, Shaul YD, Pacold ME, Kim D, Birsoy K, Sethumadhavan S, Woo HK, Jang HG, Jha AK, et al: Functional genomics reveal that the serine synthesis pathway is essential in breast cancer. Nature 476: 346-350, 2011.

14. Rhodes DR, Yu J, Shanker K, Deshpande N, Varambally R, Ghosh D, Barrette T, Pandey A and Chinnaiyan AM: ONCOMINE: A cancer microarray database and integrated data-mining platform. Neoplasia 6: 1-6, 2004. 
15. Fang Y, Fu D, Tang W, Cai Y, Ma D, Wang H, Xue R, Liu T, Huang X, Dong L, et al: Ubiquitin C-terminal Hydrolase 37, a novel predictor for hepatocellular carcinoma recurrence, promotes cell migration and invasion via interacting and deubiquitinating PRP19. Biochim Biophys Acta 1833: 559-572, 2013.

16. Jariwala U, Prescott J, Jia L, Barski A, Pregizer S, Cogan JP, Arasheben A, Tilley WD, Scher HI, Gerald WL, et al: Identification of novel androgen receptor target genes in prostate cancer. Mol Cancer 6: 39, 2007.

17. Livak KJ and Schmittgen TD: Analysis of relative gene expression data using real-time quantitative PCR and the 2(-Delta Delta $\mathrm{C}(\mathrm{T})$ ) method. Methods 25: 402-408, 2001.

18. Musgrove EA: Cyclins: Roles in mitogenic signaling and oncogenic transformation. Growth Factors 24: 13-19, 2006.

19. De Ingeniis J, Kazanov MD, Shatalin K, Gelfand MS Osterman AL and Sorci L: Glutamine versus ammonia utilization in the NAD synthetase family. PLoS One 7: e39115, 2012.

20. Mohamed A, Deng X, Khuri FR and Owonikoko TK: Altered glutamine metabolism and therapeutic opportunities for lung cancer. Clin Lung Cancer 15: 7-15, 2014.

21. De Ingeniis J, Ratnikov B, Richardson AD, Scott DA, Aza-Blanc P, De SK, Kazanov M, Pellecchia M, Ronai Z, Osterman AL and Smith JW: Functional specialization in proline biosynthesis of melanoma. PLoS One 7: e45190, 2012.

22. Ernst T, Hergenhahn M, Kenzelmann M, Cohen CD, Bonrouhi M, Weninger A, Klären R, Gröne EF, Wiesel M, Güdemann C, et al: Decrease and gain of gene expression are equally discriminatory markers for prostate carcinoma: A gene expression analysis on total and microdissected prostate tissue. Am J Pathol 160 2169-2180, 2002

23. Liu W, Hancock CN, Fischer JW, Harman M and Phang JM: Proline biosynthesis augments tumor cell growth and aerobic glycolysis: Involvement of pyridine nucleotides. Sci Rep 5: 17206, 2015.

24. Bretones G, Delgado MD and Leon J: Myc and cell cycle control. Biochim Biophys Acta 1849: 506-516, 2015.

25. McMahon SB: MYC and the control of apoptosis. Cold Spring Harb Perspect Med 4: a014407, 2014.
26. Morrish F and Hockenbery D: MYC and mitochondrial biogenesis. Cold Spring Harb Perspect Med 4: pii: a014225, 2014.

27. Liu W, Le A, Hancock C, Lane AN, Dang CV, Fan TW and Phang JM: Reprogramming of proline and glutamine metabolism contributes to the proliferative and metabolic responses regulated by oncogenic transcription factor c-MYC. Proc Natl Acad Sci USA 109: 8983-8988, 2012.

28. Kato JY, Matsuoka M, Strom DK and Sherr CJ: Regulation of cyclin D-dependent kinase 4 (cdk4) by cdk4-activating kinase. Mol Cell Biol 14: 2713-2721, 1994.

29. Han EK, Ng SC, Arber N, Begemann M and Weinstein IB: Roles of cyclin D1 and related genes in growth inhibition, senescence and apoptosis. Apoptosis 4: 213-219, 1999.

30. Gautschi O, Ratschiller D, Gugger M, Betticher DC and Heighway J: Cyclin D1 in non-small cell lung cancer: A key driver of malignant transformation. Lung Cancer 55: 1-14, 2007.

31. Biliran H Jr, Wang Y, Banerjee S, Xu H, Heng H, Thakur A, Bollig A, Sarkar FH and Liao JD: Overexpression of cyclin D1 promotes tumor cell growth and confers resistance to cisplatin-mediated apoptosis in an elastase-myc transgene-expressing pancreatic tumor cell line. Clin Cancer Res 11: 6075-6086, 2005.

32. Adams JM and Cory S: The Bcl-2 protein family: Arbiters of cell survival. Science 281: 1322-1326, 1998

33. Anagnostou VK, Lowery FJ, Zolota V, Tzelepi V, Gopinath A, Liceaga C, Panagopoulos N, Frangia K, Tanoue L, Boffa D, et al: High expression of $\mathrm{BCL}-2$ predicts favorable outcome in non-small cell lung cancer patients with non squamous histology. BMC Cancer 10: 186, 2010

This work is licensed under a Creative Commons Attribution-NonCommercial-NoDerivatives 4.0 International (CC BY-NC-ND 4.0) License. 